\title{
Space Vector Method for Voltage Dips and Swells Analysis
}

\author{
Vanya Ignatova, Pierre Granjon, and Seddik Bacha
}

\begin{abstract}
A new method for voltage dips and swells analysis is presented in this paper. This method is based on the space vector representation in the complex plane and the zero-sequence voltage. Indeed, in the case of nonfaulted system voltages, the space vector follows a circle in the complex plane with a radius equal to the nominal voltage. It follows the same shape for balanced dips, but with a smaller radius. For unbalanced dips, this shape becomes an ellipse with parameters depending on the phase(s) in drop, dip magnitude and phase angle shift. For swells the space vector shape is not modified, though the zero-sequence voltage presents significant changes in its phase and magnitude and can be used for swells analysis. The changes in the space vector and the zero-sequence voltage are used to determine the dip/swell time occurrence, to classify and finally characterize the measured power-quality disturbance. Algorithms are developed for each step of this automatic voltage dips and swells analysis (segmentation, classification, and characterization) and are validated on real measurement data.
\end{abstract}

Index Terms-Power-quality (PQ) monitoring, space vector, voltage dips (sags), voltage swells.

\section{INTRODUCTION}

V OLTAGE dips and swells are the most common types of power-quality (PQ) disturbances. They represent a major concern for the industry because they lead to important economical losses and/or distorted quality of industrial products. Indeed, equipment used in industrial plants has become more sensitive to such phenomena as a result of technology improvement and increased use of power electronics devices [1], [2]. Thus, automatic analysis of voltage dips and swells has become an essential requirement for $\mathrm{PQ}$ assessment.

Voltage dips being the most frequent PQ disturbance, the main research interests are focused on their analysis. The time duration where the dip occurs is determined by segmentation algorithms [3] applied to the three phase voltages. The dip signature can be identified from measured voltage waveforms [4], from the comparison between RMS values of phase voltages and phase-to-phase voltages [5] or well from symmetrical components [6]. Although the number and the variety of these techniques is important, none of them provides a complete voltage dips classification. Moreover, none analysis method has been developed with regard to voltage swells according to the authors' knowledge.

Manuscript received March 24, 2006; revised September 13, 2006. Current version published September 23, 2009. Paper no. TPWRD-00160-2006.

V. Ignatova is with Schneider Electric, Montbonnot St. Martin 38330, France.

P. Granjon is with the Gipsa-lab, St. Martin d'Heres 38402, France.

S. Bacha is with the G2E-lab, St. Martin d'Heres 38402, France.

Color versions of one or more of the figures in this paper are available online at http://ieeexplore.ieee.org.

Digital Object Identifier 10.1109/TPWRD.2009.2028787
In this paper a new method for joint voltage dips and swells automatic analysis is developed. It is based on space vector and zero-sequence voltage analysis, which are either used for segmenting the recorded voltage waveforms, extracting characteristic features of the dip/swell, determining its signature or evaluating its severity. The main advantages of this method are its ability to analyze either voltage dips or swells in a unified manner, its ability to provide a complete dip/swell classification, and the use of a reduced number of variables at each stage of the analysis.

This paper is organized as follows. Section II deals with voltage dips and swells signatures as a function of fault type and location, system grounding and monitor's connection. Section III describes the space vector transformation and its representation in the complex plane in case of dips and swells. Algorithms for dips and swells segmentation, classification and characterization are presented in Section IV and illustrated with examples of real measurement data.

\section{DiPS AND Swells Signatures}

Voltage sags and swells are characterized by their duration, magnitude and phase angle shift. The last two parameters determine their phasors' relation, which is also called dip/swell type or signature.

\section{A. Parameters Determining Sags and Swells Signatures}

Sags and swells signatures depend on several parameters: fault type, fault location, system grounding and connection of monitors. The influence of these parameters on the sags and swells signatures is explained in this paragraph.

1) Fault Type: There are four major types of faults: single phase-to-ground, double phase-to-ground, double phase-to-phase and three phase.

Single phase-to-ground faults are the most common fault type. They are characterized by a drop in one of the phases; the other two phases remain unchanged or increase as a function of system grounding. Double phase-to-ground faults are in the origin of major drops in two of the phases with or without phase angle shift as a function of system grounding. The voltage in the nonfaulted phase remains unchanged or increase. Double phase-to-phase faults are characterized with drops and phase angle shifts of two of the phases. The nonfaulted phase does not change. Three phase faults are the most severe fault type. They are characterized by equal drops on the three phase voltages without phase angle shift.

2) System Grounding: The system grounding affects the magnitudes of the nonfaulted phases [7]. In ungrounded or high-impedance grounded systems, voltages rise in the nonfaulted phases at the fault location. In grounded or low 

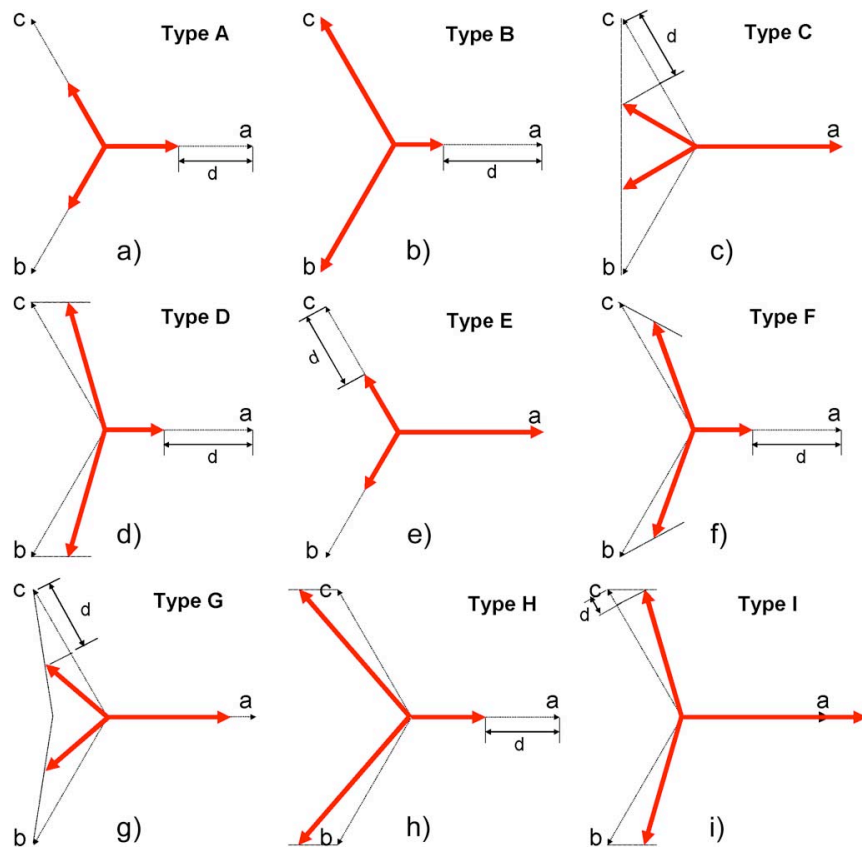

e)
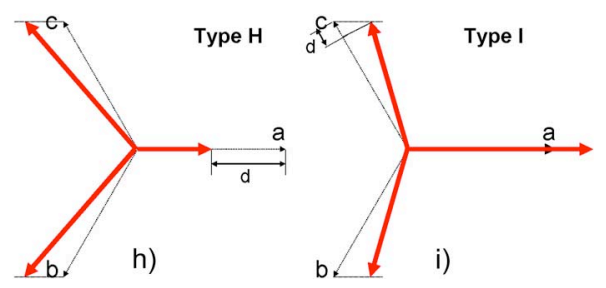

Fig. 1. Voltage dips and swells signatures.

impedance grounded systems, the nonfaulted phases remain unchanged.

3) Fault Location: Transformers usually remove the zerosequence voltage [8] and in this way they change the signature of the PQ disturbance. Therefore, voltage dips propagate down to the network changing their phasors' relation as a function of the transformer type. Swells caused by faults do not propagate; they are measured only at the same voltage level, where the fault occurred.

4) Connection of Monitors: Monitors can be connected either between phase and neutral (star connection) or between phases (delta connection) [9], [10]. Phase-to-phase connection of monitors is usually used in systems with delta connecting loads since they measure PQ disturbances as experienced by the loads. However, concerning systems with either star or delta connecting loads, phase-to-neutral measurements are recommended. Star connection of monitors provides more information about the system, for example the fault number and location. Another reason to choose star connection is that phase to phase voltages can be deduced from phase to neutral voltages, while the contrary is not possible.

\section{B. Signatures}

The most usual voltage dips signatures are presented in Fig. 1. The first seven types of voltage dips are defined in [11] and are denoted with letters from A to $\mathrm{G}$.

Voltage dips A, B, and E result from respectively three-phase, single-phase, and double-phase to ground faults, measured at the fault location. Dip C may result from phase-to-phase fault measured at the fault location, or well is obtained from the propagation of dip D via a transformer. Dips D and F are obtained from the propagation of dips $\mathrm{C}$ and $\mathrm{E}$ respectively via transformers or well are provided from phase-to-phase measurements of dips B and E at the fault location. Dip G usually results from the propagation of dip $\mathrm{F}$ [2].

In this paper, two other voltage dips types characterized with rise of the voltage in the nonfaulted phase(s) are presented and denoted with letters $\mathrm{H}$ and I. They are provided from phase to neutral measurements at the fault location in an ungrounded system and can be considered either as dips or swells. Type $\mathrm{H}$ is caused by phase to ground fault and type I is caused by double phase to ground fault.

From the presented voltage dips, four of them are characterized with major drop in one of the phases (B, D, F, H). They are called single phase voltage dips. Four dips are characterized with major drops in two of the phases and are called double phase dips (C, E, G, I). The dip type A is called a three-phase dip.

\section{Space Vector Characteristics for SAGS AND SWELLS ANALYSIS}

In this section, space vectors are defined, and their representation in the complex plane is described. Then, space vector characteristics and zero-sequence voltage are theoretically evaluated for normal system operation conditions and in the case of voltage dips/swells. It is proven that each voltage dip type is characterized by specific features, which are further used for voltage dips segmentation, classification and characterization.

\section{A. Space Vector Definition}

Space vector transformation can be directly derived from the Clarke transformation [12], defined as

$$
\left(\begin{array}{l}
x_{\alpha}(t) \\
x_{\beta}(t) \\
x_{0}(t)
\end{array}\right)=\frac{2}{3}\left(\begin{array}{ccc}
1 & -\frac{1}{2} & -\frac{1}{2} \\
0 & \frac{\sqrt{3}}{2} & -\frac{\sqrt{3}}{2} \\
\frac{1}{2} & \frac{1}{2} & \frac{1}{2}
\end{array}\right)\left(\begin{array}{l}
v_{a}(t) \\
v_{b}(t) \\
v_{c}(t)
\end{array}\right)
$$

where the coefficient $2 / 3$ is used to conserve magnitudes between the two coordinate systems.

The first two components of the Clarke transformation form the space vector (2) and the third one represents the zero-sequence voltage (3)

$$
\begin{aligned}
\vec{x}(t) & =x_{\alpha}(t)+j x_{\beta}(t)=\frac{2}{3}\left[\begin{array}{lll}
1 & e^{j \frac{2 \pi}{3}} & e^{j \frac{4 \pi}{3}}
\end{array}\right]\left[\begin{array}{l}
v_{a}(t) \\
v_{b}(t) \\
v_{c}(t)
\end{array}\right] \\
x_{0}(t) & =\frac{1}{3}\left(v_{a}(t)+v_{b}(t)+v_{c}(t)\right) .
\end{aligned}
$$

In terms of the first harmonic, the three system voltages can be viewed as sinusoidal quantities before, during and after the fault. Under this assumption and using Euler's formula, they can be represented as the sum of two contra rotating phasors with angular frequency $\omega$. For example

$$
v_{a}(t)=V \cos (\omega t+\varphi)=\frac{V}{2}\left(e^{j(\omega t+\varphi)}+e^{-j(\omega t+\varphi)}\right) .
$$

The space vector is derived from the previous voltages and consequently, can be described as the sum of positive and negative angular frequency phasors

$$
\vec{x}=x_{p} e^{j \omega t}+x_{n} e^{-j \omega t}
$$



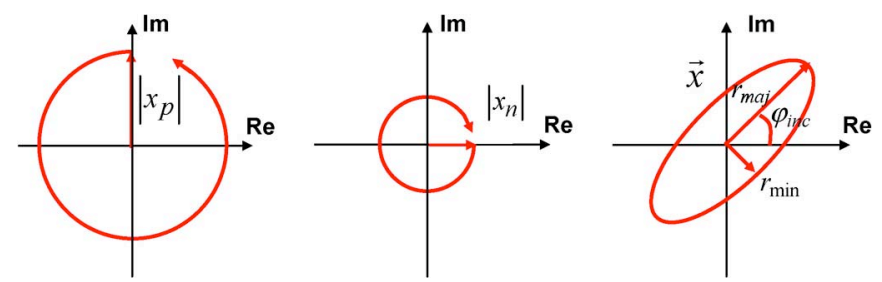

Fig. 2. Representation of the space vector as the sum of two contra rotating phasors with positive and negative angular frequency, different magnitudes, and initial phases.

where $x_{p}$ and $x_{n}$ are complex numbers

$$
x_{p}=\left|x_{p}\right| e^{j \varphi_{p}} \quad \text { and } \quad x_{n}=\left|x_{n}\right| e^{j \varphi_{n}} .
$$

When magnitudes and initial phases of positive and negative angular frequency phasors are different, the corresponding space vector follows an ellipse shape in the complex plane (Fig. 2). Major axis $r_{\text {maj }}$, minor axis $r_{\min }$, and inclination angle $\varphi_{\text {inc }}$ of this ellipse depend on previous phasors magnitudes and phases [13]

$r_{\text {maj }}=\left|x_{p}\right|+\left|x_{n}\right| \quad r_{\min }=|| x_{p}|-| x_{n} \| \quad \varphi_{\text {inc }}=\frac{1}{2}\left(\varphi_{p}+\varphi_{n}\right)$.

Note that if positive and negative angular frequency rotating phasors have the same magnitudes, $r_{\min }=0$ and the space vector is a straight line in the complex plane. Moreover, if the magnitude of one of these phasors is zero, $r_{\min }=r_{\text {maj }}$, and the space vector becomes a circle.

In order to easily characterize the shape followed by the space vector in the complex plane, a shape index is introduced as follows:

$$
S I=\frac{r_{\min }}{r_{m a j}}=\frac{|| x_{p}|-| x_{n}||}{\left|x_{p}\right|+\left|x_{n}\right|} .
$$

Its value indicates the correlation coefficient of the space vector shape to a circle:

$$
\begin{aligned}
& S I=1 \text { : circle; } \\
& 0<S I<1 \text { : ellipse; } \\
& S I=0 \text { : straight line. }
\end{aligned}
$$

\section{B. Space Vector in Case of Sags and Swells}

In a balanced sinusoidal three-phase system, the three voltages have same magnitudes and their relative phase angle shift is $2 \pi / 3$. As a result, the space vector is only composed of a positive angular frequency phasor, and represents a circle with radius equal to the nominal voltage in the complex plane. Voltage dips lead to changes in the space vector form. This paragraph demonstrates that for a three phase balanced dip, the space vector follows a circle with a radius smaller than the nominal voltage. On the contrary, the space vector is composed of positive and negative angular frequency phasors for unbalanced dips, and then follows an ellipse in the complex plane. The different types of unbalanced dips can be distinguished by using the ellipse parameters and the zero-sequence voltage. In the particular case of dips with rise of the voltage in the not faulted phase(s), the space vector follows a circle in the complex plane with radius equal to the nominal voltage, as in the

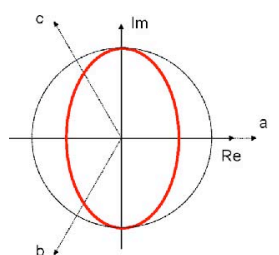

a)

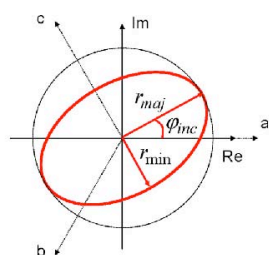

b)

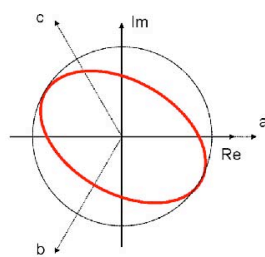

c)

\begin{tabular}{|c|c|c|c|c|c|}
\hline \multirow{2}{*}{ Type } & \multicolumn{4}{|c|}{ Space vector } & \multirow{2}{*}{$\begin{array}{c}\text { Zero sequence } \\
\text { Voltage }\end{array}$} \\
\hline & $S I$ & $\varphi_{\text {inc }}$ & $r_{\text {min }}$ & $r_{\text {maj }}$ & \\
\hline B & $1-\frac{2}{3} d$ & $\frac{5 \pi}{6}-n \frac{\pi}{3}$ & $\left(1-\frac{2}{3} d\right) V$ & V & $-\frac{d}{3} V \cos \left(\omega t+\varphi-(n-1) \frac{2 \pi}{3}\right)$ \\
\hline D & $1-d$ & $\frac{5 \pi}{6}-n \frac{\pi}{3}$ & $(1-d) V$ & $V$ & 0 \\
\hline $\mathbf{F}$ & $\frac{3(1-d)}{3-d}$ & $\frac{5 \pi}{6}-n \frac{\pi}{3}$ & $(1-d) V$ & $\left(1-\frac{d}{3}\right) V$ & 0 \\
\hline
\end{tabular}

Fig. 3. Space vectors for a single phase dip type B on phase a, b, and c, respectively.

TABLE I

Single-PHASE VOLTAGE DiPS CHARACTERISTICS

case of nonfaulted systems. In this case, only the zero-sequence voltage is used for voltage dips analysis.

The space vector and zero-sequence voltage characteristics for all types of voltage dips are determined in this paragraph. Dips are divided into single-phase, double-phase, and threephase, and swells are treated separately.

1) Single Phase Voltage Dips: Single phase dip types without swells are B, D, and F. It is demonstrated in [14] that their space vector is composed of positive and negative angular frequency phasors and describes an ellipse in the complex plane (Fig. 3). Its characteristics and zero-sequence voltage are presented in Table I, where $d$ is the dip depth and $n$ denotes the phase affected by the fault.

The ellipse inclination depends on the phase with major drop: $\varphi_{\text {inc }}=\pi / 2$ for a drop on phase a [Fig. 3(a)], $\varphi_{\text {inc }}=\pi / 6$ for a drop on phase b [Fig. 3(b)], and $\varphi_{\text {inc }}=5 \pi / 6$ for a drop on phase c [Fig. 3(c)].

The differentiation between the three single phase voltage dips is realized by the zero-sequence voltage and the ellipse major axis. Indeed, dip type B is the only one for which $x_{0} \neq 0$, and dip types $\mathrm{D}$ and $\mathrm{F}$ can be distinguished by using the ellipse major axis $\left(r_{m a j}=V\right.$ for dip type D and $r_{m a j}<V$ for dip type F).

2) Double Phase Voltage Dips: The three main double phase dip types are C, E and G. Their corresponding space vector characteristics and zero-sequence voltage are given in Table II, where $n$ denotes the phase not affected by the fault. Note that for dip types $\mathrm{C}$ and $\mathrm{G}$, the variable $d$ is a function of the phase angle shift and the drop of the phases in fault, and does not exactly represent the dip depth. However, for small phases drops, $d$ can be considered as identical to the dip depth.

For the three double phase voltage dips, the space vector is represented by a sum of two contra rotating phasors, and describes an ellipse in the complex plane with inclination angle: $\varphi_{\text {inc }}=0$ for voltage drops on phases $\mathrm{b}$ and $\mathrm{c}, \varphi_{\mathrm{inc}}=\pi / 3$ for drops on phases $\mathrm{a}$ and $\mathrm{b}$ and $\varphi_{\text {inc }}=2 \pi / 3$ for drops on phases a and c. Table II shows that the three types of double phase dips 
TABLE II

Double Phase Voltage Dips Characteristics

\begin{tabular}{|c|c|c|c|c|c|}
\hline \multirow{2}{*}{ Type } & \multicolumn{4}{|c|}{ Space vector } & Zero sequence voltage \\
\cline { 2 - 6 } & $S I$ & $\varphi_{\text {inc }}$ & $r_{\text {min }}$ & $r_{\text {maj }}$ & \\
\hline C & $1-\frac{4}{3} d$ & $(1-n) \frac{\pi}{3}$ & $\left(1-\frac{4}{3} d\right)^{\prime} V$ & $V$ & 0 \\
\hline E & $\frac{3(1-d)}{3-d}$ & $(1-n) \frac{\pi}{3}$ & $(1-d) V$ & $\left(1-\frac{d}{3}\right)_{V}$ & $\frac{d}{3} V \cos \left(\omega t+\varphi-(n-1) \frac{2 \pi}{3}\right)$ \\
\hline G & $\frac{5-6 d}{5-2 d}$ & $(1-n) \frac{\pi}{3}$ & $\left(1-\frac{6}{5} d\right) V$ & $\left(1-\frac{2}{5} d\right)^{V}$ & 0 \\
\hline
\end{tabular}

TABLE III

THREe Phase Voltage Dip CHARACTERISTICS

\begin{tabular}{|c|c|c|c|c|c|}
\hline \multirow{2}{*}{ Type } & \multicolumn{4}{|c|}{ Space vector } & Zero sequence voltage \\
\cline { 2 - 6 } & $S I$ & $\varphi_{\text {inc }}$ & $r_{\text {min }}$ & $r_{\text {maj }}$ & \\
\hline $\mathbf{A}$ & 1 & - & $(1-d) V$ & $(1-d) V$ & 0 \\
\hline
\end{tabular}

can be distinguished thanks to two parameters. The first one is the zero-sequence voltage, which is different from zero for dip type E only. The other parameter is the ellipse major axis, which is equal to the nominal voltage for the dip type $\mathrm{C}$ and inferior to the nominal voltage for the other two dip types.

3) Three Phase Voltage Dips: The space vector in the case of three phase voltage dips is only composed of one positive frequency phasor: $\vec{x}(t)=(1-d) V e^{j(\omega t+\varphi)}$. It follows a circle shape $(S I=1)$, with radius depending on the dip severity $\left(r_{\min }=r_{m a j}=(1-d) V\right)$. This dip characteristics are presented in Table III.

4) Voltage Dips With Rise in the Nonfaulted Phases: Voltage dip types $\mathrm{H}$ and I are also characterized with rise in the nonfaulted phase. For a dip type $\mathrm{H}$ the voltage change along the real axis only. For a dip type I two change directions are observed: for $0 \leq d \leq V / 4$ voltages change only along the real axis and the dip is denoted as $\mathrm{I}^{*}$; for $(V / 4) \leq d \leq V$ voltages change first along the real axis and then along the imaginary axis, the dip is denoted as $I^{* *}$. The two cases are considered separately since they do not have the same characteristics.

The space vector for voltage dip types $\mathrm{H}_{\text {and }} \mathrm{I}^{*}$ follows a circle in the complex plane with radius equal to the nominal voltage, as in the nonfaulted case. These dips can be classified and characterized thanks to the magnitude and initial phase of the zero-sequence voltage. For the dip type $I^{* *}$, the space vector follows an ellipse shape with inclination $(1-n)(\pi / 3)$, where $n$ denotes the phase not affected by the fault. It can be differentiate from dip types $\mathrm{C}, \mathrm{E}$, and $\mathrm{G}$ thanks to the magnitude of the zerosequence voltage and the ellipse major axis.

5) Conclusion: It has been shown in this section that space vector and zero-sequence voltage are useful to discriminate and identify voltage dips and swells. It can further be noted that the ellipse minor axis $r_{\min }$ and the magnitude of the zero-sequence voltage can be used to detect the occurrence of a voltage dip. Indeed, the ellipse minor axis is smaller than the nominal voltage for all dips types from A to $\mathrm{G}$, and the zero-sequence voltage has an important value for dips $\mathrm{H}$ and I. These two characteristics will therefore be used for the segmentation of recorded voltage waveforms, and the determination of the dip time area. They can also be useful to evaluate the severity of the voltage dip since

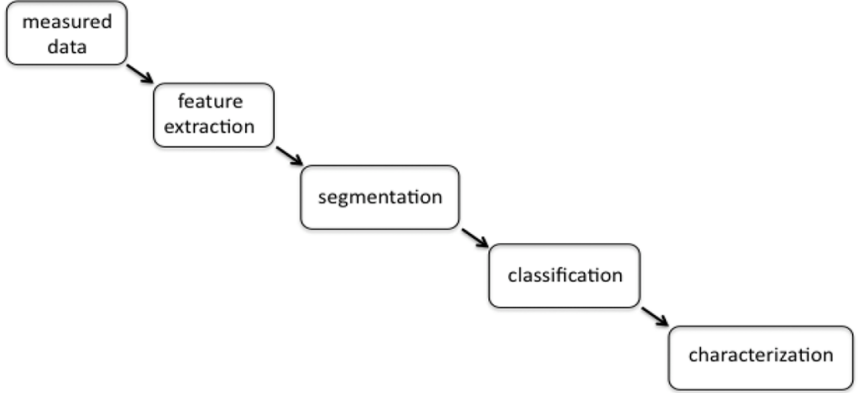

Fig. 4. Steps of voltage dips analysis.

they directly depend on the dip depth $\mathrm{d}$. The previous results and remarks will be used in the following section to develop algorithms for dips and swells segmentation, classification and characterization, and finally elaborate a complete method for voltage dips and swells analysis.

\section{Method For Sags and Swells ANalysis}

The voltage dips analysis can be considered as composed of several steps: feature extraction, segmentation, classification and characterization (Fig. 4).

In the feature extraction, the key information from the space vector and the zero-sequence voltage is captured and supplied as input of the other steps. In the next stage, the recorded voltage waveforms are segmented and the area where the dip occurs is determined. This segment is further analyzed and the dip/swell signature is estimated. At the last stage of voltage dip analysis, the severity of the measured PQ disturbance is evaluated.

In the following paragraphs, each of these steps will be explained in detail.

\section{A. Feature Extraction}

At this step, the space vector characteristics and the zerosequence voltage are evaluated.

The space vector characteristics can be directly determined by using the space vector shape in the complex plane. However, this direct estimation leads to inaccuracy in the voltage dips analysis, as measured voltages are often disturbed (noise, harmonics) and the space vector projection in the complex plane is not always characterized by an ideal shape.

This phenomenon can be illustrated with the example of the measured dip voltages in Fig. 5(a)., which present a harmonic distortion. The corresponding space vector represents a disturbed ellipse in the complex plane [Fig. 5(b)]; its spectrum [Fig. 5(c)] confirms the presence of small harmonics and noise.

The negative impact of additive noise and harmonic distortion can be minimized by applying the discrete Fourier transform (DFT) to the space vector. The complex values given by the space vector Fourier transform at $\pm 50 \mathrm{~Hz}$ correspond to positive and negative fundamental angular frequency phasors $x_{p}$ and $x_{n}$ defined in (5). Their magnitudes and phases are then used to calculate space vector characteristics $r_{\text {maj }}, r_{\text {min }}$, and $\varphi_{\text {inc }}$.

In the same way, the phase and the magnitude of the zerosequence voltage are determined by the value of its DFT at $\pm 50 \mathrm{~Hz}$ 

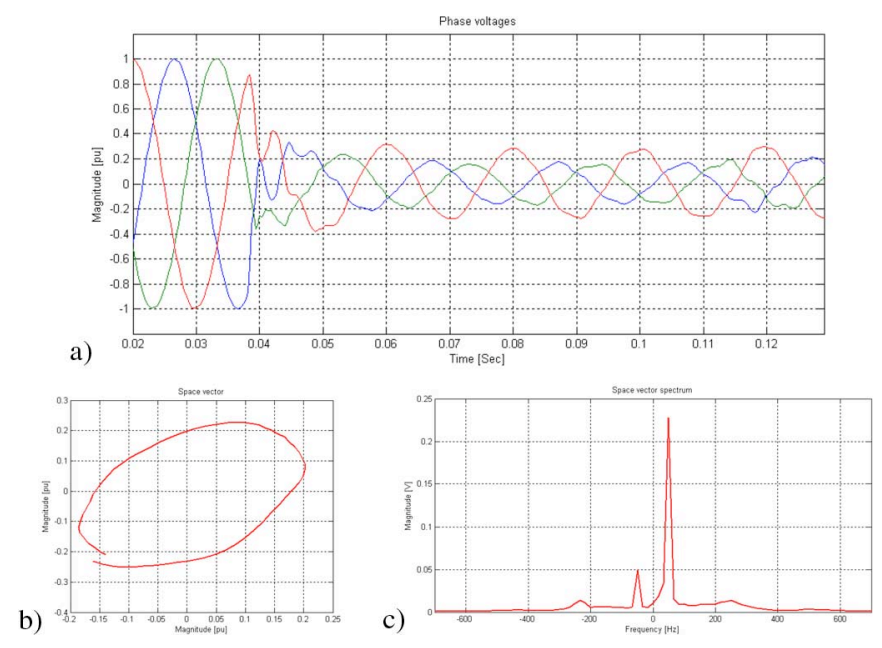

Fig. 5. (a) Measured voltage dip, (b) corresponding space vector in the complex plane, and (c) space vector spectrum.

Both variables (space vector and zero-sequence voltage) are evaluated at each sample of the recorded voltage waveforms by using one-cycle sliding window, shifted across the time axis.

\section{B. Segmentation}

The segmentation process refers to the decomposition of a given signal into stationary or weakly non stationary segments. It is a common problem in many engineering fields: speech processing, image processing, biomedical engineering and many others.

In the case of three phase voltage dips, one segmentation algorithm is usually applied to each of the three phases, because the phases changed during the dip are not known a priori. Afterwards, the results obtained on each phase have to be compared in order to make a segmentation decision for the recorded voltage waveforms.

In this section, an algorithm for voltage dips detection and segmentation based only on two variables is proposed. The first variable is the ellipse minor axis, which is equal to the nominal voltage during normal operating conditions and less than the nominal voltage for dip types from A to $\mathrm{G}$. The second variable is the zero-sequence voltage used for the segmentation of dip types $\mathrm{H}$ and I. This variable is equal or close to zero in normal system conditions and not equal to zero in case of dip types $\mathrm{H}$ and I.

The algorithm for voltage dip segmentation is graphically presented in Fig. 6.

First, the ellipse minor axis is estimated from real data as explained previously. Then, changes in its mean value are detected by the two-sided Cusum (Cumulative Sum) algorithm [15], chosen thanks to its good performance, great simplicity and optimal properties. This algorithm automatically determines different segments during which $r_{\min }$ can be considered has a constant. These constant values are estimated by averaging $r_{\min }$ on each of these segments, and compared to a threshold of 0.9 p.u. in order to determine the segments, where voltage dips occur.

If there is no dip detected thanks to $r_{\min }$, the same approach is applied to the magnitude of the zero-sequence voltage $\left|x_{0}\right|$ in

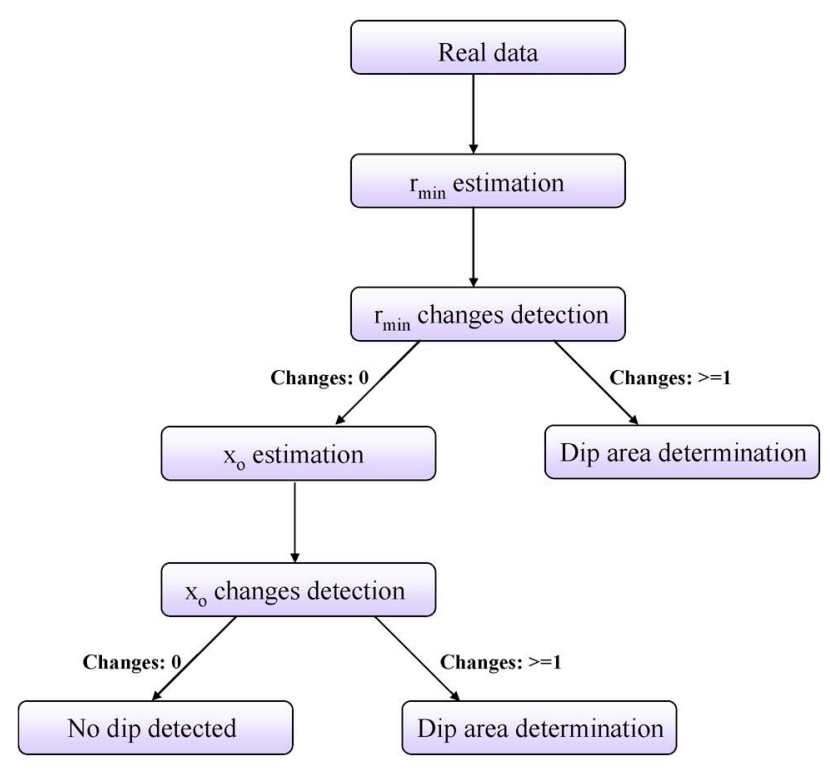

Fig. 6. Algorithm for voltage dips segmentation.

TABLE IV

CHARACTERISTICS OF Dips With SWELlS

\begin{tabular}{|c|c|c|c|c|c|}
\hline \multirow{2}{*}{ Type } & \multicolumn{4}{|c|}{ Space vector } & Zero sequence voltage \\
\cline { 2 - 6 } & $S I$ & $\varphi_{\text {inc }}$ & $r_{\text {min }}$ & $r_{\text {maj }}$ & \\
\hline H & 1 & - & $V$ & $V$ & $-d V \cos \left(\omega t+\varphi-(n-1) \frac{2 \pi}{3}\right)$ \\
\hline $\mathbf{I}^{*}$ & 1 & - & $V$ & $V$ & $2 d V \cos \left(\omega t+\varphi-(n-1) \frac{2 \pi}{3}\right)$ \\
\hline $\mathbf{I}^{* *}$ & $\frac{4}{3}(1-d)$ & $(1-n) \frac{\pi}{3}$ & $\frac{4}{3}(1-d) V$ & $V$ & $\frac{V}{2} \cos \left(\omega t+\varphi-(n-1) \frac{2 \pi}{3}\right)$ \\
\hline
\end{tabular}

order to detect eventual dips of type H or I. In this case, such a voltage dip is detected during segments where $\left|x_{0}\right|$ is greater than a small threshold chosen to be 0.1 p.u. (see Table IV with $d=0.1$ ).

\section{Classification}

The previous algorithm automatically determines the different time segments where voltage dips occur. The present one has to establish which type of dip occurs during each of these segments.

Space vector characteristics together with the zero-sequence voltage are used to classify the voltage dip type as explained in Section III. The algorithm for voltage dips classification is presented in Fig. 7. First, the space vector is evaluated and the positive and the negative fundamental frequency component $x_{p}$ and $x_{n}$ are determined by using the discrete Fourier transform as explained in Section IV-A. Then, the shape index $S I$ defined in (7) is calculated and used in order to differentiate unbalanced dips and swells from balanced ones. The limit value of the shape index is estimated for a dip depth equal to $10 \%$ of the nominal voltage (see Tables I and II with $d=0.1$ ) and is set to 0.93 .

If the dip is classified as unbalanced, the ellipse axis and inclination are calculated from the space vector phasors [see (6)].

The ellipse inclination determines whether the dip is single phase or double phase and indicates the phase(s) with major drop as shown in Fig. 8. Single phase dips are denoted with S, 


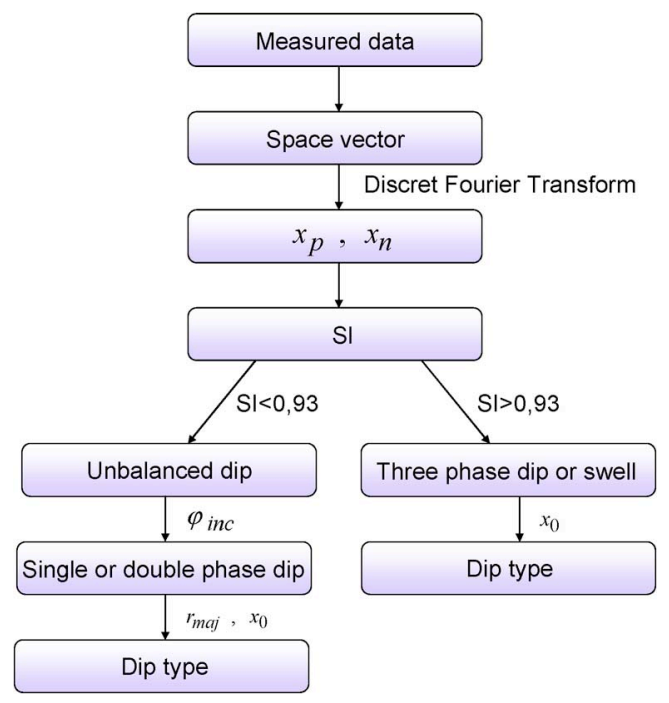

Fig. 7. Algorithm for voltage dips classification.

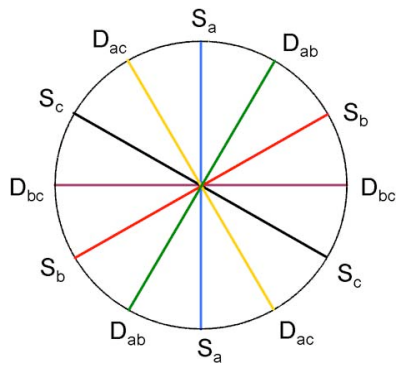

Fig. 8. Ellipse inclination angle for unbalanced dip.

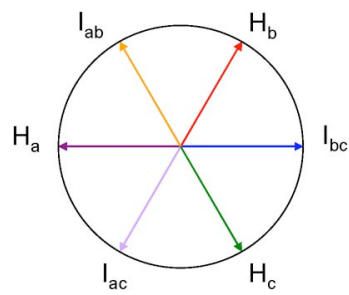

Fig. 9. Dip type as a function of the zero-sequence voltage phase.

double phase dips with $\mathrm{D}$, and phase(s) in drop are in lower case letters.

The zero-sequence voltage and the ellipse major axis contribute to the complete dip classification, differentiating dip types with the same ellipse inclination.

If the measured PQ disturbance is classified as balanced dip or swell, the zero-sequence voltage is used for further discrimination. If its magnitude is equal or close to 0 , the dip is classified as three phase balanced dip. An important value indicates that the measured signal is characterized with rise of the nonfaulted phases. Furthermore, the phase of the zero-sequence voltage indicates whether the dip type is $\mathrm{H}$ or I and which phases are affected by the fault as shown in Fig. 9.

\section{Characterization}

Once the different dips classified, their severity can be evaluated from the space vector and the zero-sequence voltage char-

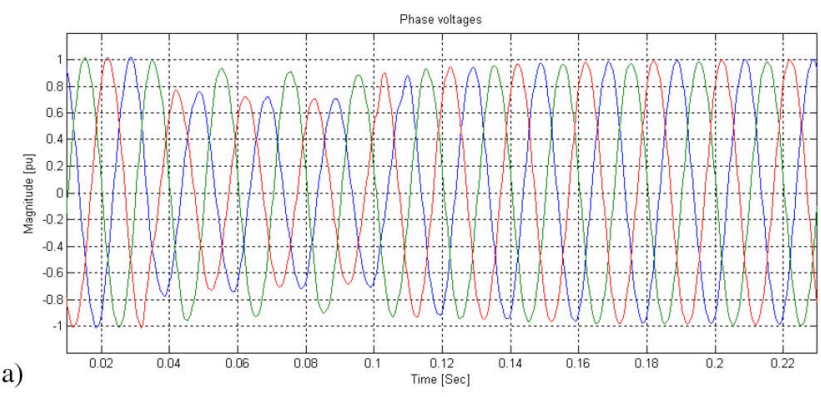

a)

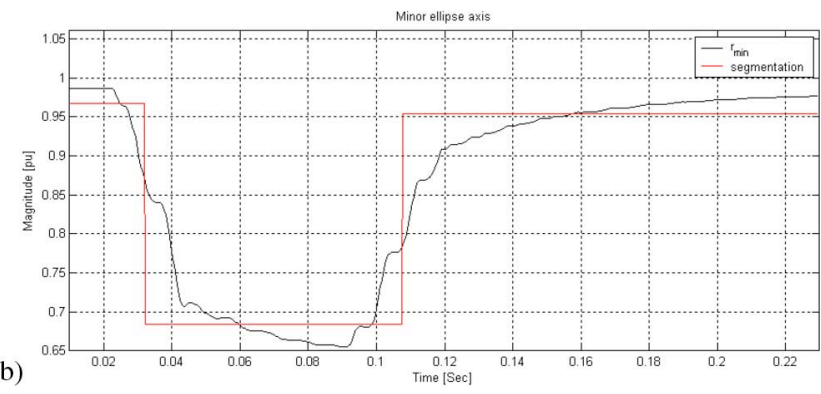

Fig. 10. (a) Double phase measured voltage dip and (b) ellipse minor axis and its segmentation.

acteristics. For voltage dips types from A to G, dip gravity can be evaluated by the ellipse minor axis $r_{\min }$ depending directly on the dip depth $d$ (see Fig. 1 and Tables I, II and III). For dip types $\mathrm{H}$ and I, the magnitude of the zero-sequence voltage $\left|x_{0}\right|$ can be used since it is proportional either to the faulted phase(s) drop, or to the nonfaulted phase(s) rise (see Fig. 1 and Table IV).

Another possibility to evaluate the gravity of measured PQ disturbances is to estimate the surface of the space vector in the complex plane. The decrease of the space vector surface is proportional to the dip gravity. For example, for a given dip depth, three phase voltage dips are the most severe, followed by double phase and single phase dips. The dips characterized with swells have the same surface as in normal system operation conditions and are not considered as severe. This corresponds to the reality, because dips of types $\mathrm{H}$ and I do not propagate down to the network and are not experienced by loads. Indeed, they are characteristic of the medium voltage level, where loads are delta connected.

\section{E. Applications}

The previous method of voltage dips analysis has been implemented in Matlab software. Its performance are illustrated in this section through results obtained on different measurement data performed on a medium voltage network. Only dips with duration over one cycle are analyzed. Phase voltages, zerosequence voltage, and all space vector characteristics are given in p.u.

The proposed method is first applied to the recorded voltage waveforms presented in Fig. 10(a). Two changes are detected in $r_{\text {min }}$ [see Fig. 10(b)] and the recorded signal is decomposed into three segments. During the second segment, the mean value of $r_{\min }$ is under the threshold of $0.9 \mathrm{pu}$ and is further analyzed. During this segment, the space vector is composed of a positive frequency phasor $x_{p}=0.78 e^{-j 158^{\circ}}$ and a negative frequency phasor $x_{n}=0.1 e^{j 38^{\circ}}$, whose complex values are estimated by 


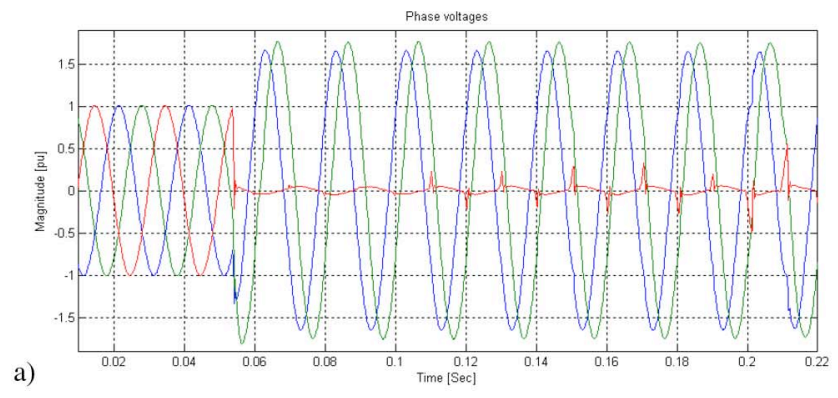

a)

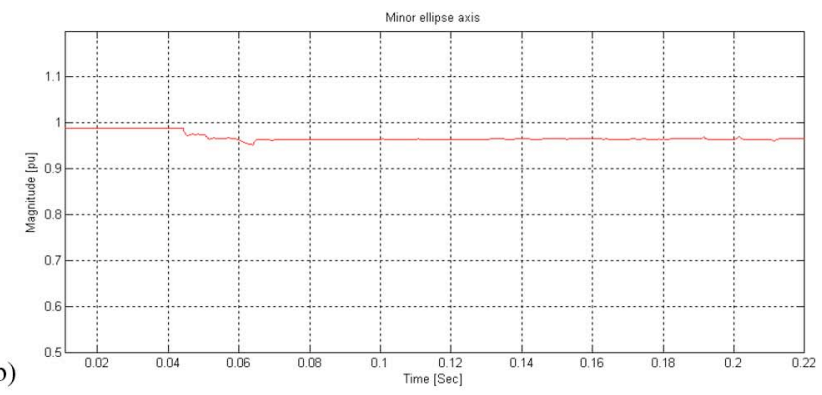

b)

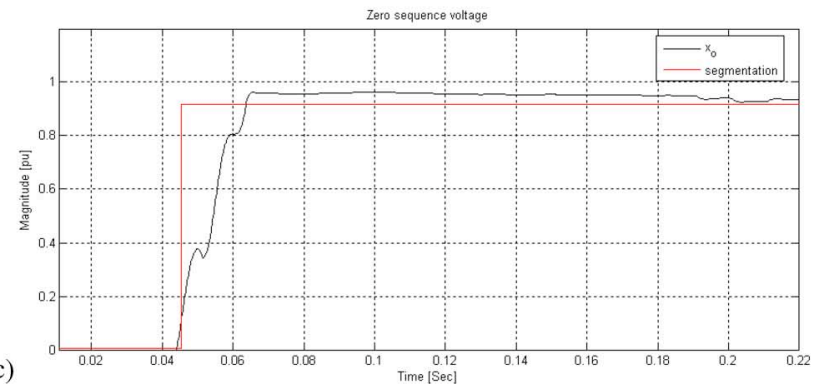

c)

Fig. 11. (a) Measured voltage dip, (b) ellipse minor axis, and (c) zero-sequence voltage and its segmentation.

the discrete Fourier transform. The shape index, estimated from the previous values, is $S I=0.76$ and classifies the dip as unbalanced (see Fig. 7). The ellipse inclination $\varphi_{\text {inc }}=120^{\circ}$ indicates that the phases $a$ and $c$ are in drop (see Fig. 8). The ellipse major axis $r_{m a j}=0.88 \mathrm{pu}$ and the zero-sequence voltage magnitude $\left|x_{0}\right|=0.04 \mathrm{pu}$ determine the dip type as $\mathrm{G}$ (see Table II). Finally, the ellipse minor axis being $r_{\min }=0.67 \mathrm{pu}$, the dip depth is evaluated at $d=0.33 \mathrm{pu}$.

The second example is the voltage dip presented in Fig. 11(a), which is characterized by a rise of the voltage in the nonfaulted phases. There is no detected change in the ellipse minor axis [see Fig. 11(b)], and therefore the zero-sequence voltage magnitude is used for dip segmentation. One change is detected in its value and the measured signal is decomposed into two segments [see Fig. 11(c)]. During the second segment, $\left|x_{0}\right|$ is greater than the threshold of $0.1 \mathrm{pu}$ and is further analyzed. The space vector and the zero-sequence voltage are estimated over one cycle window. The positive and negative fundamental frequency phasors are obtained thanks to the space vector DFT, and the shape index is estimated to $S I=0.96$. The measured PQ disturbance indicates that the dip is balanced or with a rise in the nonfaulted phases since $S I>0.93$ (see Fig. 7). Finally, the zero-sequence voltage phasor $x_{0}=0.96 e^{-j 35^{\circ}}$, also estimated thanks to DFT, classifies the dip as type $\mathrm{H}$ with a drop on the phase $c$ and a dip depth of $d=0.96 \mathrm{pu}$ (see Table IV and Fig. 9).
The recorded voltage waveforms presented in Fig. 11(a) present a high harmonic disturbance after $t=0.12$ seconds. It can be noted that even under such conditions, the estimated characteristics $r_{\min }$ and $\left|x_{0}\right|$ keep constant values and the dip type is correctly classified and characterized.

\section{CONCLUSION}

A new method for voltage dips and swells automatic detection and analysis is proposed in this paper. It is based on space vector and zero-sequence voltage characteristics, which are used either for dip segmentation, classification and characterization.

This method, able to analyze either voltage dips or swells in a unified manner, reaches very good performance even in the presence of wide band noise and/or harmonic distortion. This particularity was illustrated thanks to measurement data, and the obtained results have proved its accuracy.

Furthermore, with the help of some additional algorithms, the proposed method could be used for real-time implementation in view of on-line PQ assessment.

\section{ACKNOWLEDGMENT}

The authors gratefully acknowledge F. Dumas from Schneider Electric for his contribution with real measured data.

\section{REFERENCES}

[1] M. McGranaghan, D. Mueller, and M. Samotyj, "Voltage sags in industrial systems," IEEE Trans. Ind. Appl., vol. 29, no. 2, pp. 397-403, Mar./Apr. 1993.

[2] T. Andersson and D. Nilsson, "Test and evaluation of voltage dip immunity" Sweden, 2002, STRI Rep.

[3] E. Styvaktakis, "Automating power quality analysis," Ph.D. dissertation, Chalmers Univ. of Technol., Gothenburg, Sweden, 2002.

[4] M. H. J. Bollen and L. D. Zhang, "Different methods for classification of three-phase unbalanced voltage dips due to faults," Elect. Power Syst. Res., vol. 66, no. 1, pp. 59-69, Jul. 2003.

[5] M. H. J. Bollen and S. Styvaktakis, "Characterization of three-phase unbalanced sags, as easy as one, two, three," presented at the IEEE Power Eng. Soc. Summer Meeting, Seattle, WA, Jul. 2000.

[6] M. Bollen and L. Zhang, "A method for characterization of three phase unbalanced dips from recorded voltage waveshapes," in Proc. IEEE Telecommunication Energy Conf., Jun. 1999, p. 93.

[7] P. Heine and M. Lehtonen, "Voltage sag distributions caused by power system faults," IEEE Trans. Power Del. , vol. 18, no. 4, pp. 1367-1373, Nov. 2003.

[8] L. Zhang, "Three-phase unbalance of voltage dips," Licentiate thesis, Dept. Elect. Power Eng., Chalmers Univ. Technol., Gothenburg, Sweden, Nov. 1999.

[9] M. Didden, E. de Jaeger, W. D'haeseleer, and R. Belmans, "How to connect a voltage sag-measuring device: Phase to phase or phase to neutral?," IEEE Trans. Power Del., vol. 20, no. 2, pp. 1174-1181, Apr. 2005.

[10] R. Leborgne, G. Olguin, and M. Bollen, "The influence of pq-monitor connection on voltage dip measurement," in Inst. Elect. Eng.-MedPower, Cyprus, Nov. 2004.

[11] M. Bollen, Understanding Power Quality Problems: Voltage Sags and Interruptions. New York: Wiley-IEEE Press, 1999.

[12] J. Aller, A. Bueno, and T. Paga, "Power system analysis using space vector transformation," IEEE Trans. Power Syst., vol. 17, no. 4, pp. 957-965, Nov. 2002.

[13] N. Bachschmid, P. Pennacchi, and A. Vania, "Diagnostic significance of orbit shape analysis and its application to improve machine fault detection,” J. Braz. Soc. Mech. Sci. \& Eng., vol. 26, no. 2, Apr./Jun. 2004, Rio de Janeiro, Brazil.

[14] V. Ignatova, P. Granjon, S. Bacha, and F. Dumas, "Classification and characterization of three phase voltage dips by space vector methodology," presented at the FPS Conf., 2005.

[15] M. Basseville and I. Nikiforov, Detection of Abrupt Changes.-Theory and Application. Englewood Cliffs, NJ: Prentice-Hall, Apr. 1993. 


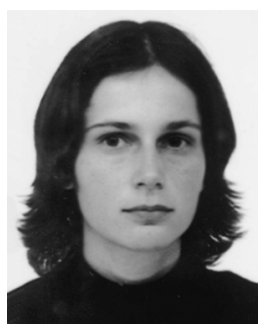

Vanya Ignatova was born in Sofia, Bulgaria, in 1979. She received the Master's degree from the National Polytechnic Institute, Grenoble, France, in 2003 and the Ph.D. degree from Joseph Fourier University, Grenoble, in 2006.

Currently, she is an expert in electrical engineering with Schneider Electric, France, where she works on software for sizing and calculation of low-voltage installations.

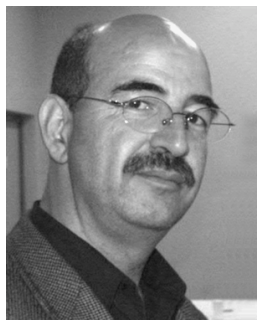

integration.
Seddik Bacha received the Eng. and Master's degrees from the National Polytechnic Institute of Algiers, Algiers, France, in 1982 and 1990, respectively, and the Ph.D. and HDR degrees from the Laboratory of Electrical Engineering of Grenoble (LEG), Grenoble, France, in 1993 and 1998, respectively.

$\mathrm{He}$ is presently Manager of the Power System Group of LEG and a Professor at the University Joseph Fourier of Grenoble. His main fields of interest are power electronics systems, modeling and control, power quality, and renewable energy

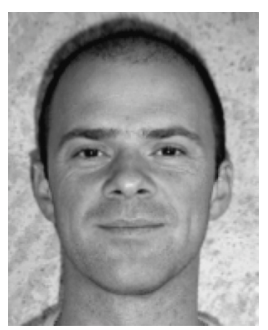

Pierre Granjon received the Ph.D. degree from the Grenoble Institute of Technology (INPG), France, in 2000.

He joined the Laboratory of Images and Signals (LIS) in 2002 and the Gipsa-lab at INPG in 2007, where he is currently Associate Professor. His current research is mainly focused on signal-processing applications in diagnosis and electrical engineering, such as fault diagnosis in electrical systems and power networks. 